L. Ein

Nagoya Math. J.

Vol. 111 (1988), 13-24

\title{
GENERALIZED NULL CORRELATION BUNDLES
}

\author{
LAWRENCE EIN
}

§ 0.

It is well known that the moduli space of stable rank 2 vector bundles on $\mathbb{P}^{2}$ of the fixed topological type is an irreducible smooth variety ([1], and [8]). There are also many known results on the classification of stable rank 2 vector bundles on $\mathbb{P}^{3}$ with "small" Chern classes.

In this paper, we investigate a particular simple class of stable rank 2 vector bundles on $\mathbb{P}^{3}$. A rank 2 vector bundle $\mathscr{E}$ on $\mathbb{P}^{3}$ is said to be a generalized null correlation bundle if it is given as the homology of a monad in the following form,

$$
0 \longrightarrow \mathcal{O}_{\mathbb{P}^{3}}(-c) \longrightarrow \mathcal{O}_{\mathbb{P}^{3}}(b) \oplus \mathcal{O}_{\mathbb{P}^{3}}(a) \oplus \mathcal{O}_{\mathbb{P}^{3}}(-a) \oplus \mathcal{O}_{\mathbb{P}^{3}}(-b) \longrightarrow \mathcal{O}_{\mathbb{P}^{3}(c)}
$$

where $c>b \geq a \geq 0$. Then $c_{1}(\mathscr{E})=0$ and $c_{2}(\mathscr{E})=c^{2}-a^{2}-b^{2}$. We observe that $\mathscr{E}$ is stable if and only if $c>a+b(1.2)$. The null correlation bundle constructed in [2] is defined with $a=b=0$ and $c=1$. Assume that $t=$ $c^{2}-a^{2}-b^{2}$ where $c>a+b$. Let $M(0, t)$ be the moduli space of stable rank 2 vector bundle on $\mathbb{P}^{3}$ with $c_{1}=0$ and $c_{2}=t$. We show that $M(0, t)$ has an irreducible component $N(a, b, c)$ with the following properties: For a general point $p$ of $N(a, b, c)$ the corresponding bundle is a vector bundle defined by a monad of the form in (0.1). Furthermore, $h^{1}$ (End $\left.\mathscr{E}\right)=$ $\operatorname{dim} N(a, b, c)$. Thus $M(0, t)$ is smooth at $p$. This implies that $M(0, t)$ is reduced at the generic point of $N(a, b, c)$.

In Section 3 we give two applications to our construction

(1) Suppose that $M(0, t)=X_{1} \cup X_{2} \cdots \cup X_{m t}$ where $X_{i}$ 's are the distinct irreducible components of $M(0, t)$. We show that $\limsup _{t} m_{t}=\infty$.

(2) We prove that $M(-1,2 n)$ is disconnected if $n \geq 2$. If $c_{1}=0$, then one can use the Atiyah-Rees invariant to show that $M(0, n)$ is disconnected for $n \geq 3$.

Throughout the paper we shall assume that ground field $k$ is algebraically

Received April 7, 1986.

Partially supported by an NSF Grant 
closed and Char $k \neq 2$.

One can show that $\mathscr{E}$ is a null correlation bundle if and only if $H^{1}(\mathscr{E}(*))$ is generated by a single element (1.3). But it should be mentioned that in general this is not a Zariski open property (e.g. $0 \rightarrow P \rightarrow$ $2 \mathcal{O}_{\mathbb{P}^{2}}(1) \oplus \mathcal{O}_{\mathbb{P}^{2}}(2) \rightarrow \mathcal{O}_{\mathbb{P}^{2}}(4) \rightarrow 0$ defines a stable rank 2 bundle with $c_{1}=0$ and $c_{2}=5$ on $\mathbb{P}^{2}$ such that $H^{1}(P(*))$ is generated by a single element of degree -4 . A general rank 2 bundle $Q$ with these chern classes can be defined by an exact sequence,

$$
0 \longrightarrow Q \longrightarrow 4 \mathcal{O}_{\mathbb{P}^{2}}(-2) \oplus \mathcal{O}_{\mathbb{P}^{2}}(-1) \longrightarrow 3 \mathcal{O}_{\mathbb{P}^{2}}(-3) \longrightarrow 0 .
$$

Observe that $H^{1}(Q(-4))=0$ and $H^{1}\left(Q\left(^{*}\right)\right)$ is generated by 3 elements of degree -3

$\S 1$.

We denote by $\mathcal{O}(t)$ the line bundle $\mathcal{O}_{\mathbb{P}^{3}}(t)$. Suppose that $c>b \geq a \geq 0$ are three nonnetative integers. Set $\mathscr{H}=\mathcal{O}(b) \oplus \mathcal{O}(a) \oplus \mathcal{O}(-a) \oplus \mathcal{O}(-b)$. Suppose that $\alpha: \mathscr{H} \rightarrow \mathcal{O}(c)$ is a surjection. Then $\mathscr{F}=\operatorname{ker}(\alpha)$ is a rank 3 vector bundle. Suppose that $\phi \in \operatorname{Hom}(\mathcal{O}(-c), \mathscr{F})$ is a general element. Observe that $\mathscr{E}={ }_{\text {def }} \operatorname{cok}(\phi)$ is a rank 2 vector bundle. Consider the following diagram:

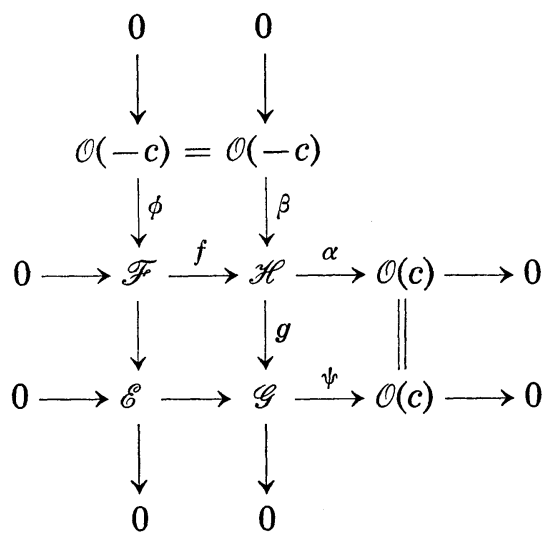

It follows that $\mathscr{E}$ is the homology of the following monad,

$$
\mathcal{O}(-c) \stackrel{\beta}{\longrightarrow} \mathscr{H} \stackrel{\alpha}{\longrightarrow} \mathcal{O}(c) \text {. }
$$

Definition. A rank 2 vector bundle $\mathscr{E}$ on $\mathbb{P}^{3}$ is said to be a generalized null correlation bundle if it can be constructed as above. 
As in [3], we can prove that 1.1.B is isomorphic to a monad of the following form:

$$
\mathcal{O}(-c) \longrightarrow \stackrel{\left(f_{4}, f_{3},-f_{2},-f_{1}\right)^{T}}{\longrightarrow} \mathscr{H} \stackrel{\left(f_{1}, f_{2}, f_{3}, f_{4}\right)}{\longrightarrow} \mathcal{O}(c)
$$

where $f_{1} \in H^{\circ}(\mathcal{O}(c-b)), f_{2} \in H^{0}(\mathcal{O}(c-a)), f_{3} \in H^{0}(\mathcal{O}(c+a))$, and $f_{4} \in H^{\circ}(\mathcal{O}(c+b))$. Let $S$ be the polynomial ring $k\left[x_{1}, x_{2}, x_{3}, x_{4}\right]$. Set

$$
M=S /\left\langle f_{1}, f_{2}, f_{3}, f_{4}\right\rangle .
$$

Observe that $M$ is a 0 -dimensional graded Gorenstein ring. Denote by $M_{j}$ the degree $j$ homogenous component of $M$. It is well known that

$$
\operatorname{dim} M_{4 c-4}=1 .
$$

Also the pairing given by the multiplication

$$
\mu_{j}: M_{j} \times M_{4 c-4-j} \longrightarrow M_{4 c-4} \cong k
$$

is perfect. From 1.1.A and the Koszul complex of $M$, we obtain the following proposition.

Proposition 1.1. Suppose that $\mathscr{E}$ is a null correlation bundle defined by the monad

$$
\mathcal{O}(-c) \longrightarrow \stackrel{\left(f_{4}, f_{3},-f_{2},-f_{1}\right)^{T}}{\longrightarrow} \mathscr{H} \stackrel{\left(f_{1}, f_{2}, f_{3}, f_{4}\right)}{\longrightarrow} \mathcal{O}(c)
$$

Then

(a) $H^{1}(\mathscr{E}(*))={ }_{\text {def }} \oplus_{j} H^{1}(\mathscr{E}(j))$ is isomorphic to $M \otimes S(c)$.

(b) $H^{2}(\mathscr{E}(*)) \cong M \otimes S(3 c)$.

(c) Denote by $E, F$, and $H$ the grades $S$-modules

$\oplus_{j} H^{0}(\mathscr{E}(j)), \oplus_{j} H^{0}(\mathscr{F}(j), \quad$ and $\quad S(b) \oplus S(a) \oplus S(-a) \oplus S(-b)$

respectively. Then $H^{1}(\mathscr{E}(*)), F$, and $E$ have the following graded minimal resolutions.

$$
\begin{aligned}
& 0 \longrightarrow \wedge^{4} H \otimes S(-3 c) \longrightarrow \wedge^{3} H \otimes S(-2 c) \longrightarrow \wedge^{2} H \otimes S(-c) \\
& \longrightarrow H \stackrel{\alpha}{\longrightarrow} S(c) \longrightarrow H^{1}(\mathscr{E}(*)) \longrightarrow 0
\end{aligned}
$$

$$
\begin{aligned}
& 0 \longrightarrow \wedge^{4} H \otimes S(-3 c) \longrightarrow \wedge^{3} H \otimes S(-2 c) \longrightarrow \wedge^{2} H \otimes S(-c) \\
& \longrightarrow F \longrightarrow 0 . \\
& 0 \longrightarrow \wedge^{4} H \otimes S(-3 c) \longrightarrow \wedge^{3} H \otimes S(-2 c) \longrightarrow K_{1} \longrightarrow E \rightarrow 0 \\
& \text { where } K_{1}=\wedge^{2} H \otimes S(-c) / S(-c) .
\end{aligned}
$$


Proof. (a) Observe that $H^{1}(\mathscr{E}(*)) \cong H^{1}(\mathscr{F}(*)) \cong M \otimes S((c)$.

(b) From the Koszul complex, we observe that

$$
M \otimes S(4 c) \cong \operatorname{Ext}^{4}(M, S) \text {. }
$$

Horrocks' theorem asserts that

$$
H^{2}(\mathscr{E}(*)) \cong \operatorname{Ext}^{4}\left(H^{1}(\mathscr{E}(*)), S\right) . \quad([7], 5.2)
$$

It follows that $H^{2}(\mathscr{E}(*)) \cong M \otimes S(3 c)$.

(c) The minimal resolution of $H^{1}(\mathscr{E}(*))$ is the Koszul complex associated with the map $\alpha: H \rightarrow S(c) . \quad F$ is defined as the kernel of $\alpha$. Hence, $F$ has the minimal resolution as claimed in 1.1.2. Finally $E=F / S(-c)$. So $E$ has the minimal resolution as claimed in 1.1.3.

Proposition 1.2. (a) $\mathscr{E}$ is stable if and only if $c>a+b$.

(b) $\mathscr{F}^{*} \cong \mathscr{G}$. (See 1.1.A for the definition of $\mathscr{F}$ and $\mathscr{G}$.)

(c) $H^{1}(\mathscr{F}(*))=M \otimes S(c)$ and $H^{2}(\mathscr{F}(*))=0$.

(d) $h^{1}(\mathscr{E}(t))=h^{1}(\mathscr{E}(2 c-4-t))$.

(e) $h^{0}(\mathscr{E}$ nd $\mathscr{F})=h^{0}(\mathscr{F} \otimes \mathscr{H})+1$.

Proof. (a) This follows from 1.1.c.

(b) The monad 1.1.c is self dual. Thus $\mathscr{F}^{*}=\mathscr{G}$.

(c) This follows from the fact $\mathscr{F}=\operatorname{ker} \alpha$.

(d) Let $m_{j}=\operatorname{dim} M_{j}$ where $M_{j}$ is the degree $j$ homogeneous component of $M$. By Serre's duality $h^{1}(\mathscr{E}(t))=h^{2}(\mathscr{E}(-t-4))$. But $h^{2}(\mathscr{E}(-t-4))$ $=\operatorname{dim} M_{3 c-t-4}=h^{1}(\mathscr{E}(2 c-t-4))$.

(e) $\mathscr{G}$ is isomorphic to $\mathscr{F} *$. Hence there is the following sequence,

$$
0 \longrightarrow \mathscr{F}(-c) \stackrel{\beta}{\longrightarrow} \mathscr{H} \otimes \mathscr{F} \longrightarrow \mathscr{F} \otimes \mathscr{F}^{*}-\rightarrow 0
$$

We may assume that $\beta=\left(f_{4}, f_{3},-f_{2},-f_{1}\right)^{T} \otimes 1$. Observe that $H^{1}\left(\mathscr{F}(-c)\left(^{*}\right)\right)$ $=M$ and $f_{1} \in \operatorname{Ann}(M)$. Thus $H^{1}(\beta)=0$ and

$$
\begin{aligned}
h^{0}\left(\mathscr{F} \otimes \mathscr{F}^{*}\right) & =h^{0}(\mathscr{H} \otimes \mathscr{F})-h^{0}(\mathscr{F}(-c))+h^{1}(\mathscr{F}(-c)) \\
& =h^{0}(\mathscr{H} \otimes \mathscr{F})+1 .
\end{aligned}
$$

Proposition 1.3. (a) Suppose that $\mathscr{E}$ is a rank 2 vector bundle on $\mathbb{P}^{3}$ with $c_{1}=0$. Then $\mathscr{E}$ is a null correlation bundle if and only if $H^{1}\left(\mathscr{E}\left({ }^{*}\right)\right)$ is generated by a single nonzero element as a graded module.

(b) Suppose that $Y_{1}$ and $Y_{2}$ are complete intersection curves of the types $(c-b, c+b)$ and $(c-a, c+a)$ respectively. Assume that $Y_{1} \cap Y_{2}=\varnothing$. Denote by the union of $Y_{1}$ and $Y_{2}$. Suppose that $\mathscr{E}$ is the normalized rank 
2 vector bundle associated with Y by Serre's construction. Then $\mathscr{E}$ is a null correlation bundle.

Proof. (a) This follows from Rao's theorem ([9]).

(b) We observe that $H^{1}(\mathscr{E}(*)) \cong H^{1}\left(I_{Y}(c)\left(^{*}\right)\right)$ is generated by a single element. Hence $\mathscr{E}$ is a null correlation bundle by (a).

Remark. Conversely, if $\mathscr{E}$ is a generalized null correlation bundle, and $s \in H^{0}(\mathscr{E}(c))$ is a general section, one can show that $(s)_{0}$ is a disjoint union of two complete intersection curves. But we do not need this result in this paper.

Set $N_{1}=\mathcal{O}(a) \oplus \mathcal{O}(-a)$ and $N_{2}=\mathcal{O}(b) \oplus \mathcal{O}(-b)$. Observe that $\mathscr{H}=N_{1}$ $\oplus N_{2}$. Suppose that $a, b$, and $c$ are integers satisfying the properties $c>b \geq a \geq 0$ and $c>a+b$. Set $t=c^{2}-a^{2}-b^{2}$. Denote by $M(0, t)$ the moduli space of stable rank 2 vector bundles on $\mathbb{P}^{3}$ with $c_{1}=0$ and $c_{2}=t$. Denote by $N(a, b, c)$ the irreducible subvariety of $M(0, t)$ parametrizing the null correlation bundles as described in 1.1.c.

Proposition 1.4. $\operatorname{dim} N(a, b, c) \geq h^{0}(\mathscr{H}(c))-h^{0}\left(N_{1} \otimes N_{1}\right)-h^{0}\left(N_{2} \otimes N_{2}\right)$ $+3-h^{0}\left(\wedge^{2} \mathscr{H}\right)$.

Proof. Denote by Hilb $(c-a, c+a, c-b, c+b)$ the irreducible open subset of the Hilbert scheme corresponding to the disjoint union of complete intersection curves as described in $1.3(\mathrm{~b})$. Then

$$
\begin{aligned}
\operatorname{dim} \operatorname{Hilb}(c-a, c+a, c-b, c+b) \\
\quad=h^{0}(\mathscr{H}(c))-h^{0}\left(N_{1} \otimes N_{1}^{*}\right)-h^{0}\left(N_{2} \otimes N_{2}^{*}\right) \\
\quad=\operatorname{dim} h^{0}(\mathscr{H}(c))-h^{0}\left(N_{1} \otimes N_{1}\right)-h^{0}\left(N_{2} \otimes N_{2}\right) .
\end{aligned}
$$

It follows from Theorem 1.1 of [5] and Proposition 1.3, $\operatorname{dim} N(a, b, c) \geq$ $\operatorname{dim}(\operatorname{Hilb}(c-a, c+a, c-b, c+b))+2-h^{0}(\mathscr{E}(c))$. Since $c>a+b$, it follows from (1.12) and (1.13),

$$
h^{0}(\mathscr{E}(c))=h^{0}\left(\wedge^{2} \mathscr{H}\right)-1=h^{0}(\mathscr{F}(c))-1 .
$$

$\S 2$.

In this section, we shall compute $h^{1}(\mathscr{E} n \mathscr{E})$ where $\mathscr{E}$ is a generalized null correlation bundle. Lemma 2.1 is the key to the computation. Unfortunately, the proof of 2.1 depends on a long computation using Cech cycles. So first we will assume the result in 2.1 and prove Theorem 2.2. Then we will prove 2.1 at the end of this section. 
LEMma 2.1. Let $\mathscr{E}$ and $\mathscr{G}$ be the bundles as defined in 1.1.A and 1.1.c. Then the natural map between the graded modules

$$
H^{1}(\mathscr{E} \otimes \mathscr{G}(*)) \longrightarrow H^{1}(\mathscr{E}(c)(*))
$$

is surjective.

Theorem 2.2. Suppose that $\mathscr{E}$ is a stable null correlation bundle as defined in 1.1.c. Then $h^{1}(\mathscr{E}$ nd $\mathscr{E})=\operatorname{dim} N(a, b, c)$.

Proof. It follows from 1.1.A. and Lemma 2.1 that we have the following exact sequence,

$$
\begin{aligned}
& 0 \longrightarrow H^{0}(\mathscr{E} \text { nd } \mathscr{E}) \longrightarrow H^{0}(\mathscr{E} \otimes \mathscr{G}) \longrightarrow H^{0}(\mathscr{E}(c)) \longrightarrow H^{1}(\mathscr{E} \text { nd } \mathscr{E}) \\
& \longrightarrow H^{1}(\mathscr{E} \otimes \mathscr{G}) \longrightarrow H^{1}(\mathscr{E}(c)) \longrightarrow 0
\end{aligned}
$$

Consider the exact sequence,

$$
0-\mathscr{E}(-c) \stackrel{\beta}{\longrightarrow} \mathscr{E} \otimes \mathscr{H}-\mathscr{E} \otimes \mathscr{G}-0
$$

where $\beta=1 \otimes\left(f_{4}, f_{3},-f_{2},-f_{1}\right)^{T}$. Observe that $H^{1}(\mathscr{E}(*))=M \otimes S(c)$ and $H^{2}(\mathscr{E}(*))=M \otimes S(3 c)$. This implies that both $H^{1}(\beta)$ and $H^{2}(\beta)$ are the zero map. Hence

$$
h^{1}(\mathscr{E} \otimes \mathscr{G})-h^{2}(\mathscr{E}(-c))=h^{1}(\mathscr{E} \otimes \mathscr{G})-h^{1}(\mathscr{E}(c))=h^{1}(\mathscr{E} \otimes \mathscr{H})
$$

But

$$
h^{1}(\mathscr{E} \otimes \mathscr{H})=h^{0}(\mathscr{H}(c))+h^{0}\left(\mathscr{H} \otimes \wedge^{2} \mathscr{H} \otimes \mathcal{O}(-c)\right)-h^{0}(\mathscr{H} \otimes \mathscr{H})
$$

by 1.1 .1 .

Also

$$
\begin{aligned}
h^{0}(\mathscr{E} \otimes \mathscr{G}) & =h^{0}(\mathscr{E} \otimes \mathscr{H})+h^{1}(\mathscr{E}(-c)) \\
& =h^{0}(\mathscr{E} \otimes \mathscr{H})+1 \\
& =h^{0}\left(\mathscr{H} \otimes \wedge^{2} \mathscr{H} \otimes \mathcal{O}(-c)\right)+1 .
\end{aligned}
$$

Tensor the exact sequence (1.13) by $H$ and use the fact $a+b<c$, we obtain the last equality $h^{0}(\mathscr{E} \otimes \mathscr{H})=h^{\wedge}\left(\mathscr{H} \otimes \wedge^{2} \mathscr{H} \otimes \mathcal{O}(-c)\right)$.

$$
h^{0}(\mathscr{E}(c))=h^{0}(\mathscr{F}(c))-1=h^{0}\left(\wedge^{2} \mathscr{H}\right)-1 .
$$

It follows that

(2.2.B) $\quad h^{1}(\mathscr{E}$ nd $\mathscr{E})=h^{0}(\mathscr{E}(c))+\left[h^{1}(\mathscr{E} \otimes \mathscr{G})-h^{1}(\mathscr{E}(c))\right]-h^{0}(\mathscr{E} \otimes \mathscr{G})+1$

$$
=h^{0}\left(\wedge^{2} \mathscr{H}\right)-1+h^{0}(\mathscr{H}(c))-h^{0}(\mathscr{H} \otimes \mathscr{H})
$$


Recall that $\mathscr{H}=N_{1} \oplus N_{2}$ (1.4). Observe that $h^{0}(\mathscr{H} \otimes \mathscr{H})=h^{0}\left(N_{1} \otimes N_{1}\right)$ $+h^{0}\left(N_{2} \otimes N_{2}\right)+2 h^{0}\left(N_{1} \otimes N_{2}\right)$. Also $h^{0}\left(\wedge^{2} \mathscr{H}\right)=h^{0}\left(N_{1} \otimes N_{2}\right)+2$. So we can rewrite (2.2.B)

$$
h^{1}(\mathscr{E} \text { nd } \mathscr{E})=h^{0}(\mathscr{H}(c))-h^{0}\left(N_{1} \otimes N_{1}\right)-h^{0}\left(N_{2} \otimes N_{2}\right)-h^{0}\left(\wedge^{2} \mathscr{H}\right)+3 .
$$

This implies that $h^{1}(\mathscr{E} n d \mathscr{E}) \leq \operatorname{dim} N(a, b, c)$, by 1.4. But $H^{1}(\mathscr{E} n d \mathscr{E})$ is the Zariski target space of $M(0, t)$ at the point corresponding to $\mathscr{E}$. Thus $h^{1}(\mathscr{E}$ nd $\mathscr{E}) \geq \operatorname{dim} N(a, b, c)$. Hence $h^{1}(\mathscr{E}$ nd $\mathscr{E})=\operatorname{dim} N(a, b, c)$. This completes the proof of 2.2. Next we will prove 2.1.

Proof of 2.1. $H^{1}(\mathscr{E}(*))$ is generated as a graded module by a single element in degree $-c$. In order to prove 2.1 , it will be sufficient to show $H^{1}(\mathscr{E} \otimes \mathscr{G}(-2 c))-H^{1}(\mathscr{E}(-c))$ is surjective. First we shall use Cech cohomology to construct a nonzero element $x \in H^{1}(\mathscr{E}(-c))$. Consider the following exact sequences of graded $S$-modules.

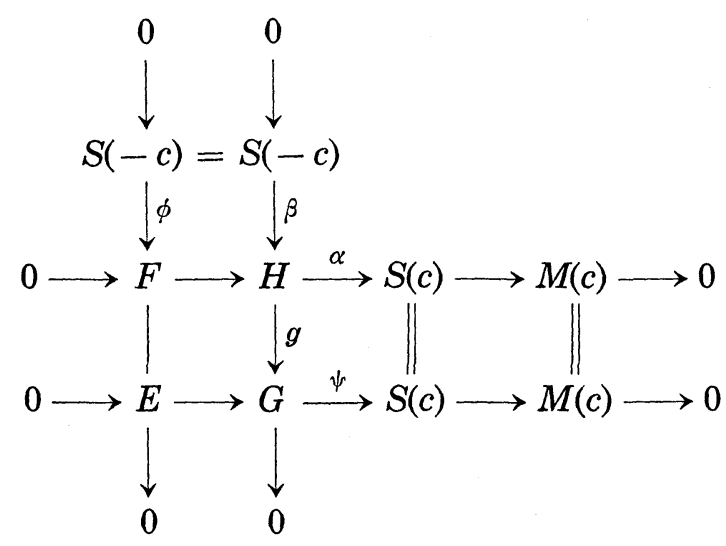

We shall assume that

$$
\beta=\left(f_{4}, f_{3},-f_{1},-f_{2}\right)^{T} \text { and } \alpha=\left(f_{1}, f_{2}, f_{3}, f_{4}\right) .
$$

Suppose that $t_{1}, t_{2}, t_{3}, t_{4}$ are the homogeneous minimal generators of $H$ such that $\alpha\left(t_{i}\right)=f_{i}$ for $i=1,2,3$ and 4. Set $e_{i}=g\left(t_{i}\right) \in G$. Observe that $e_{i}$ 's are the minimal generators of $G$. Furthermore, $e_{i}$ 's satisfy the relation

$$
f_{4} e_{1}+f_{3} e_{2}-f_{2} e_{3}-f_{1} e_{4}=0 .
$$

Let $U_{i}$ be the affine open set in $\mathbb{P}^{3}$ defined by $f_{i} \neq 0$. Consider the exact sequence

$$
H^{0}\left(\mathscr{G}(-c) \stackrel{\psi}{\longrightarrow} H^{0}(\mathcal{O}) \stackrel{\delta}{\longrightarrow} H^{1}(\mathscr{E}(-c))\right.
$$


Observe that $x=\delta(1)$ is a nonzero element in $H^{1}(\mathscr{E}(-c))$. Now $\left.\psi\right|_{U_{i}}\left(e_{i} / f_{i}\right)$ $=1$. It follows that we can give the following Cech cohomological description of $x$,

$$
\begin{aligned}
x=\delta(1)=\left(\frac{e_{i}}{f_{i}}-\frac{e_{j}}{f_{j}}\right)_{i, j} & \in \prod_{1 \leq i<j \leq 4} H^{0}\left(U_{i} \cap U_{j},\left.\mathscr{E}(-c)\right|_{U_{i} \cap U_{j}}\right) \\
& =C^{1}(U, \mathscr{G}(-c)) .
\end{aligned}
$$

Next we shall construct an element $y \in H^{1}(\mathscr{E}(-c) \otimes \mathscr{G}(-c))$ which will map onto $x$. Consider the following elements in $H^{0}\left(U_{i} \cap U_{j}, \mathscr{G}(-c) \otimes\right.$ $\mathscr{G}(-c))$

$$
\begin{aligned}
A_{i j} & =\frac{e_{j}}{f_{j}} \otimes \frac{e_{i}}{f_{i}}-\frac{e_{i}}{f_{i}} \otimes \frac{e_{j}}{f_{j}}, \\
B_{i j} & =\frac{e_{j}}{f_{j}} \otimes \frac{e_{j}}{f_{j}}-\frac{e_{i}}{f_{i}} \otimes \frac{e_{i}}{f_{i}}, \\
C_{1,4} & =\frac{f_{2} f_{3}}{f_{1} f_{4}} A_{2,3}+A_{1,4}, \\
C_{2,3} & =\frac{f_{1} f_{4}}{f_{2} f_{3}} A_{1,4}+A_{2,3}, \\
C_{1,2} & =C_{1,3}=C_{2,4}=C_{3,4}=0 .
\end{aligned}
$$

Set

$$
y=\left(A_{i, j}+B_{i, j}-C_{i, j}\right)_{j, j} \in C^{1}(U, \mathscr{G}(-c) \otimes \mathscr{G}(-c)) .
$$

Recall that the map $g: G \rightarrow S(c)$ is defined by $g\left(e_{i}\right)=f_{i}$. Also recall that $E$ is defined as the kernel of $g$. One checks easily that $g \otimes 1(y)=0$ in $C^{1}(U, \mathscr{G}(-c))$. So $y \in C^{1}(U, \mathscr{E}(-c) \otimes \mathscr{G}(-c))$. Consider the boundary operator,

$$
\begin{aligned}
\triangle: C^{1}(U, \mathscr{E}(-c) \otimes \mathscr{G}(-c)) & \longrightarrow C^{2}(U, \mathscr{E}(-c) \otimes \mathscr{G}(-c)) \\
& \left.\longrightarrow C^{2}(U, \mathscr{G}(-c)) \otimes \mathscr{G}(-c)\right) .
\end{aligned}
$$

Now

$$
C^{2}(U, \mathscr{G}(-c) \otimes \mathscr{G}(-c))=\prod_{1 \leq i<j<k \leq 4} H^{0}\left(U_{i} \cap U_{j} \cap U_{k}, \mathscr{G}(-c) \otimes \mathscr{G}(-c)\right)
$$

Set $\triangle y=\left(Z_{i, j, k}\right)$. Observe that

$$
\begin{aligned}
Z_{1,2,3} & =A_{1,2}-A_{1,3}+A_{2,3}-C_{2,3} \\
& =A_{1,2}-A_{1,3}-\frac{f_{1} f_{4}}{f_{2} f_{3}} A_{1,4} .
\end{aligned}
$$


It follows from 2.1.B.

$$
f_{4} e_{1} \otimes e_{1}+f_{3} e_{2} \otimes e_{1}-f_{2} e_{3} \otimes e_{1}-f_{1} e_{4} \otimes e_{1}=0
$$

and

$$
f_{4} e_{1} \otimes e_{1}+f_{3} e_{1} \otimes e_{2}-f_{2} e_{1} \otimes e_{3}-f_{1} e_{1} \otimes e_{4}=0
$$

Hence

$$
f_{3}\left(e_{2} \otimes e_{1}-e_{1} \otimes e_{2}\right)-f_{2}\left(e_{3} \otimes e_{1}-e_{1} \otimes e_{3}\right)-f_{1}\left(e_{4} \otimes e_{1}-e_{1} \otimes e_{4}\right)=0 .
$$

This implies that $z_{1,2,3}=0$.

Using the similar methods, we can show that $z_{1,2,4}, z_{1,3,4}$ and $z_{2,3,4}$ are also equal to zero. Thus $\triangle(y)=0$ in $C^{2}(U, \mathscr{E}(-c) \otimes \mathscr{G}(-c))$. This implies that $y \in Z^{1}(U, \mathscr{E}(-c) \otimes \mathscr{G}(-c))$. Also observe that

$$
1 \otimes g(y)=2\left(\frac{e_{j}}{f_{j}}-\frac{e_{i}}{f_{i}}\right)_{i, j}=2 \cdot x .
$$

Since $h^{1}(\mathscr{E}(-c))=1$, observe that, $H^{1}(\mathscr{E} \otimes \mathscr{G}(-2 c)) \rightarrow H^{1}(\mathscr{E}(-c))$ is surjective. This implies, that $\left.H^{1}(\mathscr{E} \otimes \mathscr{G}(-c))(*)\right) \rightarrow H^{1}(\mathscr{E}(*))$ is surjective.

$\S 3$.

Theorem 3.1. (a) The closure of $N(a, b, c)$ in $M(0, t)$ is an irreducible component of $M(0, t)$.

(b) If $p \in N(a, b, c)$, then $p$ is a smooth point of $M(0, t)$.

Proof. If $\mathscr{E}_{p}$ is the null correlation bundle corresponding to $p$, then $\operatorname{dim} \Theta_{M(0, t), p}=h^{1}\left(\mathscr{E} n d \mathscr{E}_{p}\right)=\operatorname{dim} N(a, b, c)$. It follows that the closure of $N(a, b, c)$ is an irreducible component and $p$ is a smooth point of $M(0, t)$.

Q.E.D.

So far we have only discussed null correlation bundles with $c_{1}=0$. One can define null correlation bundles with $c_{1}=-1$ by the following monad:

$$
\mathcal{O}(-c-1) \stackrel{\beta}{-} \mathcal{O}(b) \otimes \mathcal{O}(a) \otimes \mathcal{O}(-a-1) \otimes \mathcal{O}(-b-1) \stackrel{\alpha}{-} \mathcal{O}(c)
$$

where $c>b \geq a \geq 0$.

Suppose $\mathscr{E}$ is the rank 2 bundle defined as the homology of 3.1.A. Then $c_{1}(\mathscr{E})=-1$ and $c_{2}(\mathscr{E})=\left(c^{2}-a^{2}-b^{2}\right)+(c-a-b)$. Similarly we can show that $H^{1}(\mathscr{E}(*))$ is generated by a single element of degree $-c$. One can also show that $\mathscr{E}$ is stable if and only if $c>a+b$. Suppose 
that $c>a+b$. Let $t=c^{2}-a^{2}-b^{2}+c-a-b$. Denote by $M(-1, t)$ the moduli space of stable rank 2 bundles with $c_{1}=-1$ and $c_{2}=t$. Set $N^{\prime}(a, b, c)$ be the irreducible subvariety of $M(-1, t)$ parametrizing those null correlation bundles defined by a monad as in 3.1.A.

Theorem 3.2. (a) The closure of $N^{\prime}(a, b, c)$ is an irreducible component of $M(-1, t)$.

(b) If $p \in N^{\prime}(a, b, c)$, then $p$ is a smooth point of $M(-1, t)$.

Proof. The proof of 3.2, is essentially identical to the proof of 3.1. We shall leave it to the readers.

Theorem 3.3. (a) The subvariety $N(0, n, n+1)(n \geq 0)$ is a smooth irreducible component of $M(0,2 n+1)$. Furthermore, $N(0, n, n+1)$ does not intersect other irreducible components of $M(0,2 n)$.

(b) The subvariety $N^{\prime}(0, n, n+1)$ is a smooth irreducible component of $M(-1,2 n+2)$. Furthermore, $N^{\prime}(0, n, n+1)$ does not intersect other irreducible components of $M(-1,2 n)$.

Proof. (b) Denote by $\bar{N}^{\prime}(0, n, n+1)$ the closure of $N^{\prime}(0, n, n+1)$ in $M(-1,2 n)$. Let $\mathscr{E}$ be a rank 2 stable vector bundle corresponding to a point in $\bar{N}^{\prime}(0, n, n+1)$. Then $h^{1}(\mathscr{E}(-n-1)) \neq 0$ and $h^{0}(\mathscr{E}(1)) \geq 1$. Hence $\mathscr{E}$ has the maximal spectrum $([6])$. It follows that $h^{1}(\mathscr{E}(-n-1))=1$ and $h^{1}(\mathscr{E}(-n))=3$. Since $h^{1}(\mathscr{E}(-n))=3$, there is a linear form 1 such that the multiplication map, $H^{1}(\mathscr{E}(-n-1)) \rightarrow H^{1}(\mathscr{E}(-n))$ is the zero map. Let $L$ be the plane defined by 1 . Then $h^{0}\left(\mathscr{E}_{L}(-n)\right)=h^{1}(\mathscr{E}(-n-1))=1$.

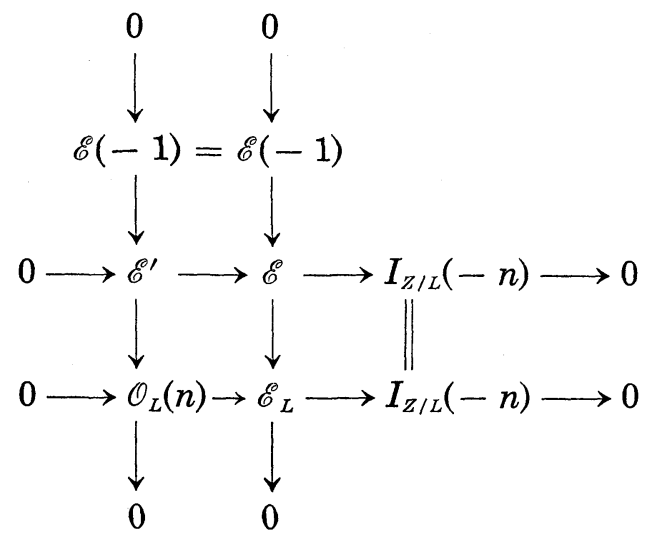

Denote by $I_{Z / L}$ the ideal sheaf of the zero set of the unique section of $\mathscr{E}_{L}(-n)$. Observe that $h^{0}\left(\mathscr{E}^{\prime}(j)\right)=0$ if $j \leq 0$ and $h^{0}(\mathscr{E}(1))=1$. It follows 
from the spectrum of $\mathscr{E}, H^{\circ}\left(\mathcal{O}_{L}(n+1+j)\right) \cong H^{1}(\mathscr{E}(j))$ for $j<0$. There is also an exact sequence

$$
0 \longrightarrow H^{0}\left(\mathscr{E}^{\prime}(1)\right) \longrightarrow H^{0}\left(\mathcal{O}_{L}(n+1)\right) \longrightarrow H^{1}(\mathscr{E}) .
$$

It follows that the graded module $H^{1}(\mathscr{E}(*))$ has only one minimal generator $x$ of negative degree. Now $\operatorname{deg} x=-n-1$. There is a linear form $l$ such that $l \cdot x=0$ and a homogeneous polynomial $f$ of degree $n+1$ such that $f \cdot x=0$. Consider the minimal presentation of $H^{1}(\mathscr{E}(*))$ as a $S$-module:

$$
L_{1} \longrightarrow L_{0} \longrightarrow H^{1}(\mathscr{E}(*)) \longrightarrow 0 .
$$

We have shown that $L_{1} \cong S(n) \oplus S \oplus \oplus_{j} S\left(a_{j}\right)$ where $a_{j}<0$. Rao's theorem asserted that $L_{1} \cong L_{1}^{\curlyvee}(-1) \quad([9], 2.3)$. Hence $L_{1}=S(n) \oplus S \oplus S(-1) \oplus$ $S(-n-1)$. Also Rao's theorem asserts that $2\left(\operatorname{rank} L_{0}\right)+2=\operatorname{rank}\left(L_{1}\right)$ ([9], 2.2). Hence rank $L_{0}=1$. It follows that $H^{1}(\mathscr{E}(*))$ is generated by $x$ and $\mathscr{E}$ is a null correlation bundle. Thus $N^{\prime}(0, n, n+1)=\bar{N}^{\prime}(0, n, n+1)$. But $h^{1}(\mathscr{E}$ nd $\mathscr{E})=\operatorname{dim} N^{\prime}(0, n, n+1)$. Hence $M(-1,2 n+2)$ is smooth along $N^{\prime}(0, n, n+1)$. Thus $N(0, n, n+1)$ does not intersect other components. The proof of (a) is similar to (b). We shall leave it to the readers.

Corollary 3.4. $M(-1,2 n)$ is disconnected if $n \geq 2$.

Remark. The subvariety $N(0, n, n+1)$ in $M(0,2 n+1)$ has already been studied by Ellingsrud and Stromme in [4]. They have proved that $\bar{N}(0, n, n+1)$ is an irreducible component by a different method.

Lemma 3.5. Let $t=p_{1} p_{2} \cdots p_{m}$ where $p_{i}$ 's are distinct positive odd numbers. Consider the set

$$
T=\left\{\begin{array}{l|l}
(a, b, c) & \begin{array}{l}
a, b, c \text { are nonnegative integers } \\
c>a+b \text { and } c^{2}-a^{2}-b^{2}=t
\end{array}
\end{array}\right\} .
$$

Then $|T| \geq m$.

Proof. Let $a_{i}=0, b_{i}=\frac{1}{2}\left(\left(t / p_{i}\right)-p_{i}\right)$, and $c_{i}=\frac{1}{2}\left(\left(t / p_{i}\right)+p_{i}\right)$. Clearly $\left(a_{i}, b_{i}, c_{i}\right) \in T$. Hence $|T| \geq m$.

Proposition 3.6. Suppose that $M(0, t)=X_{1} \cup X_{2} \cdots \cup X_{m_{t}}$ where $X_{i}$ 's are the distinct irreducible components of $M(0, t)$. Then $\limsup _{t} M_{t}=\infty$.

Proof. This follows from 3.1 and 3.5. 


\section{REFERENCES}

[1] W. Barth, Moduli of vector bundles on the projective plane, Invent. Math., 42 (1977), 63-91.

[2] — Some properties of stable rank 2 bundles on $\mathbb{P}_{n}$, Math. Ann., 226 (1977), 125-150.

[ 3 ] W. Barth and K. Hulek, Monads and moduli of vector bundles, Manuscripta Math., 25 (1978), 323-347.

[4.] G. Ellingsrud and S. Stromme, Stable vector bundles of rank 2 on $\mathbb{P}^{3}$, with $c_{1}=0$ and $c_{2}=3$, Math. Ann., 255 (1981), 123-135.

[5] R. Hartshorne, Stable vector bundles of rank 2 on $\mathbb{P}^{3}$ Math. Ann., 238 (1978), 229-280.

[6] - - Stable reflexive sheaves, Math. Ann., 254 (1980), 121-176.

[7] G. Horrocks, Vector bundles on the punctured spectrum of a local ring, Proc. London Math. Soc., 3 (1964), 689-713.

[8] M. Maruyama, Moduli of stable sheaves I, J. Math. Kyoto, 18 (1978), 557-614.

[ 9 ] P. Rao, A note on cohomology modules of rank 2 bundles, J. of Algebra, 86 (1984), 23-34.

Department of Math., Stat., Comp. Sci.

University of Illinois at Chicago

Chicago, Illinois 60680

U.S.A. 